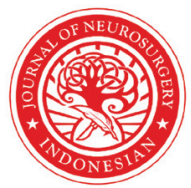

Indonesian Journal of Neurosurgery

${ }^{1}$ Medical student. Center for Biomedical

Research (CIB), Faculty of Medicine,

University of Cartagena, Cartagena,

Colombia.

${ }^{2}$ Neurosurgeon, Critical Care. Center for Biomedical Research (CIB). Director of the Cartagena Neurotrauma Research Group Research Line. Faculty of Medicine, University of Cartagena. Cartagena de Indias, Bolívar.

${ }^{3}$ Professor of Neurosurgery. University of Campinas (UNICAMP), Campinas-SP, Brazil

${ }^{4}$ Department of Neurosurgery, All India Institute of Medical Sciences, Saket

Nagar, Bhopal, Madhya Pradesh, India. ${ }^{5}$ Department of Neurosurgery, National Institute of Mental Health and Neurosciences, Bengaluru, Karnataka, India.

*Corresponding to:

Ezequiel García-Ballestas; Center for Biomedical Research (CIB), Faculty of Medicine, University of Cartagena,

Cartagena, Colombia;

mineurocirujano@aol.com

Received: 2020-12-22

Accepted: 2021-07-29

Published: 2021-08-13

\section{Stimulation of vagal nerve in intracranial hypertension: A literature review}

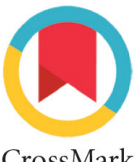

Ezequiel García-Ballestas ${ }^{1 *}$, Yeider Durango-Espinosa ${ }^{1}$, Romario Mendoza-Flórez ${ }^{1}$, Amit Agrawal ${ }^{4}$, Harsh Deora ${ }^{5}$, Liza Matallana-Ramírez' ${ }^{1}$, Andrei F. Joaquim ${ }^{3}$, Luis Rafael Moscote-Salazar ${ }^{2}$

\section{ABSTRACT}

Background: Pathologies that affect the central nervous system (CNS) and increases intracranial pressure may develop into fatal outcomes. There are physiological properties of neurons that are susceptible to modify, such as neuronal plasticity. The vagus nerve stimulation (VNS) has focused on the modification of these variables in order to restore the homeostasis of the CNS. In this article, we aimed to review the general concept of VNS, the pathology of intracranial hypertension and the effects of combining treatment to the pathology.

Literature review: VNS activates several neuromodulatory pathways and centers in the brain, which associated with plasticity. They are including cholinergic and noreadrenergic system, which are transcendental for neural plasticity. One of the mechanisms by which VNS decreases ICP is due to the attenuation of the systemic inflammatory response and the signaling of proinflammatory cells induced by TBI, whose possible mechanism is the inhibition of cytokines such as tumor necrosis factor (TNF), IL-1b, IL-6 and IL-18. In addition, it had been shown that VNS stimulated the locus coeruleus with a consequent release of noreepinephrine, which act as an endogenous anti-inflammatory agent. VNS requires neuroanatomical knowledge of the entire vagal network and its physiology. The surgery is relatively simple and the complication rates are very low conferring a great effectiveness in several neural diseases. Neural and non-neural pathways have been well-characterized to avoid the immune response through VNS.

Conclusion: Different experimental studies have concluded that VNS reduces intracranial pressure, although the mechanism is not completely specified. Further studies evaluating the clinical role of VNS in intracranial hypertension are necessary.

Keywords: intracranial hypertension, vagus nerve, vagus nerve stimulation

Cite This Article: Garcia-Balesta, E., Durango-Espinosa, Y., Mendoza-Florez, R., Agrawal, A., Deora, H., Matallana-Ramirez, L., Joaquim, A.F., Moscote-Salazar, L., . 2021. Stimulation of vagal nerve in intracranial hypertension: A literature review. Indonesian Journal of Neurosurgery 4(2): 21-26. D0I: 10.15562/ijn.v4i1.109

\section{INTRODUCTION}

Pathology in the brain leads to abnormal function of the nervous system. ${ }^{1}$ The mass effect created by certain pathologies can result in a syndrome of systemic inflammatory response, sepsis and multi organ failure., ${ }^{2,3}$ Recently, the therapy that aiming to modify plasticity (neuromodulation) had been explored. ${ }^{1}$ Neuromodulation is a therapy consisting by administering energy to the nervous system to excitate, inhibit or modify neural activity to modify the natural course of a certain disease. $^{3}$

At the end of the nineteenth century, it was known that the parasympathetic nervous system was compromised in certain diseases. In 1884, Corning performed first transcutaneous electrical stimulation of the vagus nerve to modify certain cardiovascular parameter. ${ }^{4}$ Unfortunately, it did not succed, but it was the beginning of a new era to treat pathologies of the nervous system. ${ }^{5}$ Since then, several findings had strengthened the theoretical basis of technical effects of this practice, which was used to investigate its effects on animals. ${ }^{6,7}$ In humans, it was approved in 1997 to treat epilepsy. Further, it is also approved to treat major depressive disorder and Alzheimer's disease in $2005 .^{3,5-7}$ In present time, it is performed to relieve headaches, arthritis, asthma, joint pain, fibromyalgia, irritable bowel syndrome, gastric motility disorders, obesity, hemostasis, bipolar disorder and dementia. ${ }^{5}$ It had been accepted that vagus nerve stimulation (VNS) provided beneficial effect in the treatment of intracranial hypertension using brief burst stimuli of vagus nerve ${ }^{1}$ or by implanting a subcutaneous pulse generator. ${ }^{3}$

The VNS was used by more than 50,000 people in the world. It had been postulated that VNS acted through several mechanisms such as reduction of TNF- $\alpha^{2,6}$ and proinflammatory interleukins ${ }^{3,6}$; increase noreadrenaline ${ }^{6,8}$; and etc., which still the subject of various questions and continuous research. ${ }^{6}$ In this article, we aimed to review the general concept of VNS, the pathology of intracranial hypertension and the effects of combining treatment to the pathology.

\section{INTRACRANIAL HYPERTENSION}

The skull cavity contains $70 \%$ of neural tissue, $15 \%$ of blood and $15 \%$ 
of cerebrospinal fluid (CSF), which are surrounded by dura mater and bone. The sum of the pressures exerted by each of these components is known as intracranial pressure (ICP), which varies according to age, body position and clinical condition., ${ }^{9,10}$ Brain tissue, CSF and intracranial blood have a combined volume of approximately 1,200 to $1,500 \mathrm{ml}$ and normal ICP is usually between 5 to $15 \mathrm{mmHg}$ in normal condition. ${ }^{10,11}$ Intracranial hypertension is defined as the elevation of the ICP above $20 \mathrm{mmHg}$, which sustained for at least 10 minutes. ${ }^{11,12}$ The pathophysiology of intracranial hypertension is determined by the Monroe-Kellie doctrine, which states that the total volume of intracranial contents is constant and that any increase in any of those components will initially fill a small potential space of only a few milliliters in volume. Then, it must be compensated by a decrease in the volume of another intracranial component to avoid an increase in the ICP., ${ }^{90,12}$ The CSF and intracranial venous blood move to the spinal compartment when the ICP increases due to its capability to diminishes quick and efficient. However, this compensation is limited and ICP may increases rapidly when decompensation occurs. ${ }^{911}$ There is a relationship between volume and intracranial pressure. Initially, the pressure increases slightly with the increase in volume, but when the compensation mechanisms are exceeded, the pressure increases rapidly. ${ }^{11}$

CSF is produced by choroid plexus and circulates through the ventricular system. It is subsequently absorbed by the arachnoid villi and venous granulations.
Any factor that alters the flow of CSF or its absorption in the vasculature can often lead to an increase of the ICP. ${ }^{9}$ Intracranial hypertension decreases cerebral perfusion pressure (CPP), which defined as mean arterial blood pressure (MAP) minus ICP. CPP is the driving force behind cerebral blood flow, and as it decreases, cerebral blood flow can be insufficient for adequate cerebral oxygenation. This process could induce additional cytotoxic edema and result in an even higher ICP. ${ }^{11}$

Human body has autoregulation mechanisms to maintain blood pressure between 50 and $150 \mathrm{mmHg}$, either by vasodilatation or vasoconstriction. This autoregulation mechanism maintains adequate cerebral blood flow and reduce injury to the brain parenchyma..$^{9,12}$ In addition, it is important to note that cranial vault is divided into several compartment by dural folds. Because of this, the ICP is not similar throughout the skull cavity. Increases in the contents of a region of the brain can cause regional increases in the ICP. In extreme cases, the contents of that compartment can move, or herniated, to a different compartment. ${ }^{9,11,12}$

Any condition that alters the intracranial content could cause elevations of the ICP. It is important to understand that the impact of the increase in ICP depends on the cause and the progressivity. Pressure greater than $15 \mathrm{mmHg}$ is poorly tolerated in rapid progression, as in the case of traumatic brain injury (TBI). On the other hand, increased ICP due to tumor gives better adaptation because of slow progression. ${ }^{12}$ In Table 1, we can see the main causes of intracranial hypertension.
In specific situations such as TBI, disruption of the blood-brain barrier (BBB) leads to accumulation of fluid and active molecules inside the brain parenchyma. ${ }^{13}$ This process leads to cell destruction and produces primary lesions which can lead to post-traumatic pathophysiological consequences, such as cell excitotoxicity, inflammation and cerebral edema that leads to intracranial hypertension. ${ }^{3,13}$

\section{VAGAL NERVE STIMULATION}

Vagus nerve stimulation emerges as a therapy to enhance rehabilitation of various sensory, motor and cognitive neuropathologies by inducing neuroplasticity. ${ }^{14}$ Vagus nerve was known providing neuroplasticity due to modulation of central nervous system (CNS) and direct pathway to memory function. ${ }^{1}$

The vagus nerve was originated from the brainstem to innervates different thoracoabdominal viscera and responsible for the parasympathetic function of these organs. ${ }^{4,5}$ It receives several sensory pathway to modulate anatomical and functional connections of this nerve, which explain its effects in the CNS. ${ }^{15}$ The nucleus of vagus nerve is consisted of four nuclei, which are nucleus of the solitary tract, dorsal motor nucleus of the vagus nerve, spinal nucleus of the trigeminal nerve, and ambiguus nucleus (Figure 1). ${ }^{4}$ The fibers of these nuclei converge in a single trunk and emerge from the spinal bulb to traverse the foramen jugulare. Furthermore, it is divided into several terminations (atrial,

Table 1. Main causes of intracranial hypertension ${ }^{13}$

\begin{tabular}{|c|c|c|c|}
\hline Increase in brain volume & Increase in CSF & $\begin{array}{c}\text { Increase in blood } \\
\text { volume }\end{array}$ & Mixed \\
\hline 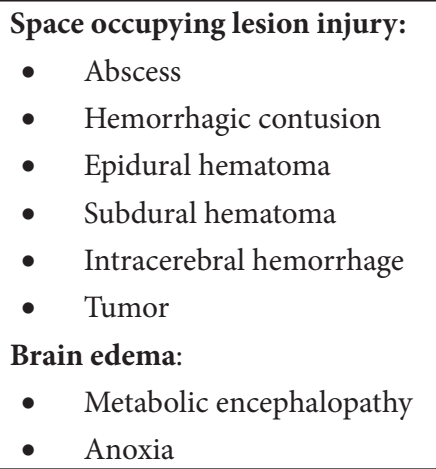 & $\begin{array}{l}\text { 1. } \text { Hydrocephalus } \\
\text { 2. Arachnoid cyst } \\
\text { 3. Choroidal plexus papilloma }\end{array}$ & $\begin{array}{ll}\text { 1. } & \text { Hyperemia } \\
\text { 2. Hypercapnia } \\
\text { 3. Obstruction of blood } \\
\text { flow }\end{array}$ & $\begin{array}{ll}\text { 1. } & \text { Arteriovenous malformations } \\
\text { 2. } & \text { Hypertensive encephalopathy } \\
\text { 3. } & \text { Traumatic brain injury } \\
\text { 4. } & \text { Subarachnoid hemorrhage } \\
\text { 5. Abdominal hypertension } \\
\text { 6. }\end{array}$ \\
\hline
\end{tabular}


meningeal, sympathetic, pharyngeal and cardiac), some of which are subdivided into nervous plexus (cardiac, pulmonary, esophageal, celiac, myenteric, adrenal, hepatic and gastroduodenal)., ${ }^{4,5}$

More than $80 \%$ of the fibers (all of which are composed of fibers A, B and, mostly C) are directed to nucleus of solitary tract. In nucleus of solitary tract, it releases excitatory neurotransmitters such as glutamate and aspartate. They have capability to inhibit $\gamma$-aminobutyric acid (GABA), acetylcholine, nitric oxide, vasoactive intestinal peptide and other neuropeptides for signal transduction. ${ }^{5}$ The efferent fibers of nucleus solitary tract to mesencephalic nuclei such as locus coeruleus and dorsal raphe magnus release norepinephrine and serotonin to entire

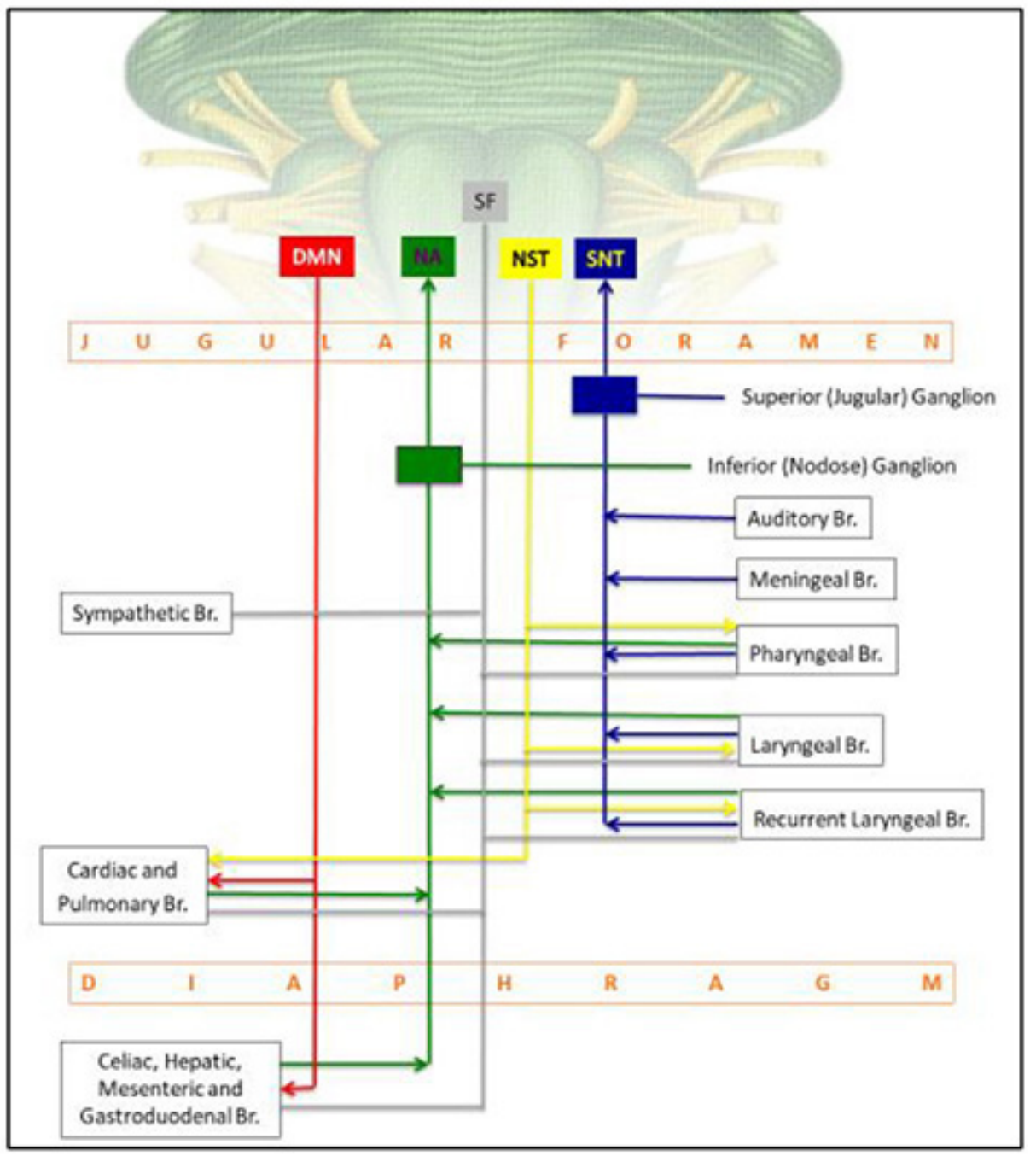

Figure 1. Vagus nerve anatomy. Each arrow shows the unidirectional conduction. DMN: dorsal motor nucleus; NA: nucleus ambiguus; SF: sympathetic fibers; NST: nucleus of the solitary tract; SNT: nucleus of the trigeminal nerve.
The right VNS is intended for the treatment of heart failure, so it will not be mentioned in this literature. ${ }^{5}$ The vagus nerve stimulation therapy equipment is composed of a programmable pulse generator, a bipolar helical electrode, a programming stick linked to a software that allows programming and evaluation, a tunneling tool and magnets. Each stimulation period is preceded by 2 seconds of acceleration time, followed by 2 seconds of reduction ramp time. ${ }^{16}$

The surgical procedure to put left cervical vagal stimulator requires general anesthesia. Incision on lateral of pectoralis major muscle is performed to put the generator. The second incision is on the neck where the electrode cable is attached to the left mid-cervical vagus nerve and thus the conductor cable passing through a subcutaneous tunnel to be connected to the generator. This is an ambulatory procedure and usually lasts from 1 to 3 hours under general anesthesia. ${ }^{17}$

The programmable parameters are the current load (intensity of the electrical stimulus, measured in miliamperes (mA)), which is $0.25 \mathrm{~mA}$ initially; the width of the pulse (duration of the electrical pulse, measured in microseconds), $250-500 \mu \mathrm{s}$ initially; the pulse frequency (measured in Hertz $(\mathrm{Hz})$ ), which is $30 \mathrm{~Hz}$ initially; and the activation/ deactivation of the working cycle (the stimulus on and off, measured in seconds or minutes). ${ }^{15}$ The optimal configuration is still unknown but the most prudent thing to do is start at low frequencies. ${ }^{5}$ By placing the programming stick on the generator, the software allows us to read and alter the stimulation parameters. ${ }^{16}$ It also contains mechanical and electrical safety features that reduce the possibility of high frequency stimulation that could cause tissue damage. ${ }^{17}$ Therefore, the moderate intensity stimulation produces a cortical motor reorganization greater than the low or high stimulation. ${ }^{14}$ The generator works continuously, but each patient is given a magnet to deactivates the stimulation if placed on the pulse generator. The generator should also be deactivated when withdrawing the normal programmed stimulation. ${ }^{5,17}$

The electrical stimulation of the vagus nerve stimulates activity in the 
anterior cholinergic basal brain and the noreadrenergic locus coeruleus, leading to the subsequent release of neuromodulators throughout the cortex. Both of neuromodulator system are essential for cortical neuroplasticity. ${ }^{1} \mathrm{VNS}$ adjusts norepinephrine levels in the brain and promotes recovery. ${ }^{6}$ It is necessary to emphasize that the activation of the locus coeruleus following VNS directly influences the cerebral cortex. Locus coeruleus innervates almost all levels of the central nervous system because it is the only sources of noreepinephrine of the cerebral and cerebellar cortices. ${ }^{4}$

Adverse events are mostly related to the surgical procedure or to the electrical stimulation itself. Adverse events related to the surgical procedure include peritracheal hematoma or vagus nerve trauma. Surgical complications are rare, but it is known that during surgery, bradycardia and asystole may occur during the guide impedance test. ${ }^{5}$ Adverse events related to stimulation include voice disturbance, cough, dyspnea, headache and paresthesia in the left cervical region. ${ }^{5,15-17}$ Long-term arrhythmias and delayed syncopes have been reported. ${ }^{5}$ Vagus nerve stimulation may have an unfavorable impact on sleep breathing disorders (obstructive sleep apnea). ${ }^{16}$

\section{SURGICAL TECHNIQUE}

The implantation of the right and left vagus nerve stimulator had shown similar efficacy. However, left side was preferred to avoid cardiovascular side effects due to innervation of the right vagus nerve to sinoatrial node. ${ }^{18}$ It is necessary to prepare atropine and catecholamines in case of bradycardia and asystole during intraoperative stimulation. ${ }^{19}$

Two incisions are made, one in the neck for the implantation of the electrodes and one in the chest for the implantation of the impulse generator. First incision is made in skinfold of the neck at midpoint between the mastoid and the clavicle 3 to $4 \mathrm{~cm}$ in length, from the midline to the medial border of the sternocleidomastoid. ${ }^{18}$ The second incision should be made a little above the axillary fold, medial to the shoulder joint. The platysma is divided and retracted, the deep cervical fascia is opened in the anterior border of the sternocleidomastoid muscle and the medial blunt dissection is performed. Identification of ansa cervicalis should be done and should not be confused with vagus nerve, subsequently dissects the loop. ${ }^{19}$ At this point, the procedure should continue using the operating microscope or the surgical loops. Dissection of the nerve of the carotid is performed with a blunt hook and then dissect the vagus nerve carefully (to preserve the vasa nervorum intact) with the help of a loop in a length of 3 to $4 \mathrm{~cm}^{18}$

First, the anchor is placed comfortably around the nerve allowing minimal movements. After that, we place the positive (central) and negative (upper) electrodes. Next, the electrode impedance is tested together with the generator function. After this, a medial subcutaneous pouch is formed to the armpit or to the breast, $5 \mathrm{~cm}$ in length and height. The stimulator cable is placed by passing the tunneling device from the subcutaneous pouch to the cervical incision, where the electrode is anchored at two points to the silicone supports in the deep cervical fascia and near the sternocleidomastoid muscle. The battery is fixed to the lead electrode and anchored by nonabsorbable sutures to the fascia of the pectoral muscle. Finally, the two wounds are closed in anatomical layers using absorbable sutures. ${ }^{18,19}$

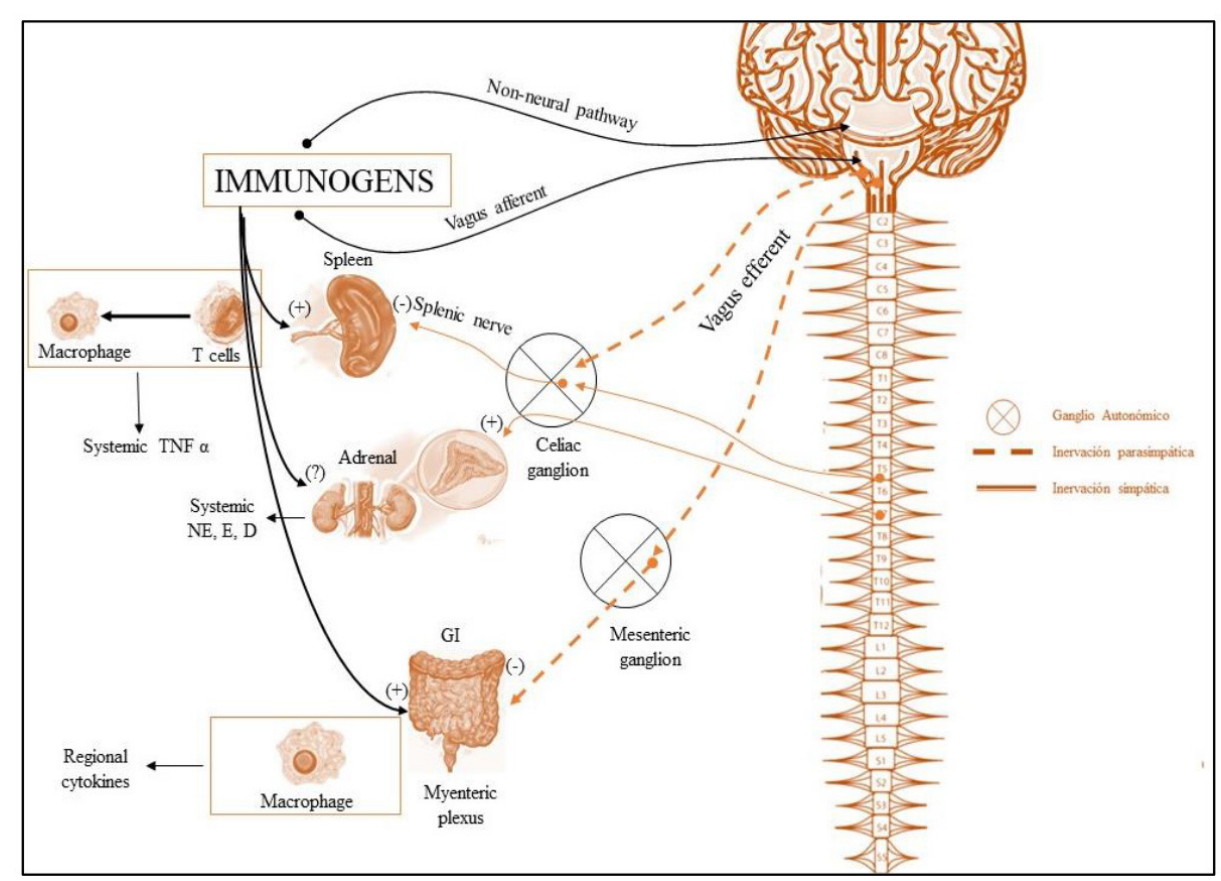

Figure 2. Different pathways of mechanisms of VNS.

\section{MECHANISM OF ACTION OF VNS}

Synaptic plasticity is the most important point of restoration following TBI, which occurs due to synaptogenesis. It is believed that VNS could enhance synaptic plasticity and provided neuroprotective effects (Figure 2). ${ }^{6,20}$ VNS activates several neuromodulatory pathways and centers in the brain, which associated with plasticity. They are including cholinergic and noreadrenergic system, which are transcendental for neural plasticity. ${ }^{20}$ One of the mechanisms by which VNS decreases ICP is due to the attenuation of the systemic inflammatory response and the signaling of proinflammatory cells induced by TBI, whose possible mechanism is the inhibition of cytokines such as tumor necrosis factor (TNF), IL$1 \mathrm{~b}$, IL-6 and IL-18. . $^{2,3,6,13,15}$ In addition, it had been shown that VNS stimulated the locus coeruleus with a consequent release of noreepinephrine, which act as an endogenous anti-inflammatory agent. ${ }^{3,6,7,15,21,22}$ Noreepinephrine also has protective role in vasogenic edema following TBI due to regulation of cerebral blood flow. ${ }^{7}$ Noreepinephrine levels also reported increase in amygdala, hippocampus, and cerebral cortex of rats following VNS. ${ }^{22}$ Furthermore, it 
had been shown that VNS increased plasma levels of ghrelin, which provided neuroprotection following brain injury indicated by reduction of cerebral edema and inflammatory cytokines. ${ }^{2,3,6,13}$ In addition, it was shown that ghrelin negatively regulated TNF. ${ }^{6}$

Endotoxemia activated immune response, which originated in the spleen. An indirect parasympathetic autonomic regulation (by preganglionic fibers of the celiac ganglia) and direct sympathetic (postganglionic fibers) induced release of TNF- $\alpha$ by the splenic macrophages. VNS does not act through this autonomic pathway, but through a non-neural peripheral pathway called the cholinergic anti-inflammatory pathway, which involves nicotinic cholinergic receptor- $\alpha 7$ ( $\alpha 7 \mathrm{nAChR})$. VNS stimulates release of acetylcholine from the celiac ganglion on the spleen, resulting in a release of noreepinephrine that interacts with $\beta 2 \mathrm{AR}$ receptor cells containing choline acetyltransferase for the synthesis of acetylcholine. They will be released and act through $\alpha 7 \mathrm{nAChR}$ in splenic macrophages and downregulate the expression of NFKB / STAT3, decreasing TNF- $\alpha$, box 1 of the high mobility group and other cytokines that are expressed before the endotoxemic stimulus.

In the case of TBI, cell excitotoxicity, inflammation, and cerebral edema may cause an increase in ICP. ${ }^{3,6}$ Patients with increased ICP following TBI are associated with a worse prognosis. ${ }^{3}$ For this reason, an important objective when treating a severe TBI is to find a way to control the ICP and cerebral perfusion in a timely manner. Neuromodulation techniques have been accepted as a treatment for various neurological and psychiatric pathologies. In recent years, VNS have been investigated as a treatment for TBI with very promising results. ${ }^{3}$

\section{EXPERIMENTAL STUDIES ADDRESSING VNS AND ICP}

Clough, et al. evaluated the ability of VNS to reduce cerebral edema in traumatic brain injured rats. ${ }^{7}$ They reported a remarkable and statistically significant decrease of edema at 2 days. Decrease of cerebral edema was determined by calculating the difference of weight between hydrated and dehydrated (in an oven at $100^{\circ} \mathrm{C}$ ) brain. The percentage of water in the ipsilateral hippocampus in control group was 80.71 \pm 0.41 , while in VNS was $80.43 \pm 0.34$ (p $<0.041$ ). However, the reduction was not significant in prefrontal cortex, cerebellum and brainstem. The reduction of edema is indirectly related to the decrease of ICP. However, the mechanism was still unclear. ${ }^{3}$

Smith et al. used a percussion model of brain lesions in rats to show that VNS was able to reduces cortical edema induced by TBI and also improves cognitive function. ${ }^{22}$ Pruitt et al. observed that combination of lower level VNS and rehabilitation training was effective for improvement. ${ }^{20}$ On the other hand, Lopez et al. demonstrated that VNS decreases the permeability of blood-brain barrier in patients with TBI. VNS inhibited high regulation of aquaporin 4 (AQP-4) that was induced by TBI, which contributed to the decrease in cerebral edema and ICP. ${ }^{13}$

Tubbs et al. experimented in pigs using VNS with stimulation parameters similar to those clinically used for the treatment of epilepsy. ${ }^{23}$ They reported that VNS (especially the left vagus nerve) produced an average reduction of $6 \mathrm{mmHg}$ in ICP within 15 to 35 minutes after stimulating the nerve. It did not cause alterations in cardiovascular responses, such as bradycardia or hypotension. ${ }^{23}$ VNS may play a role in controlling the ICP. However, it is necessary to conduct further research to determine applicable conclusions in humans regarding VNS for TBI. ${ }^{6}$

\section{CONCLUSION}

VNS requires neuroanatomical knowledge of the entire vagal network and its physiology. The surgery is relatively simple and the complication rates are very low conferring a great effectiveness in several neural diseases. Neural and non-neural pathways have been well-characterized to avoid the immune response through VNS. Different experimental studies have concluded that VNS reduces intracranial pressure, although the mechanism is not completely specified. Further studies evaluating the clinical role of VNS in intracranial hypertension are necessary.

\section{CONFLICT OF INTEREST}

There is no conflict of interest related to the materials or methods used in this study.

\section{FUNDING}

This article received no specific funding from any funding agency in the public, commercial, or not-for-profit sectors.

\section{AUTHOR CONTRIBUTIONS}

Authors took part in design of the study, contributed to data collection, participated in writing the manuscript and all agree to accept equal responsibility for accuracy of the contents of this article.

\section{REFERENCES:}

1. Hays SA. Enhancing rehabilitative therapies with vagus nerve stimulation. Neurotherapeutics. 2016; 13(2): 382 - 394. https://doi.org/10.1007/ s13311-015-0417-z.

2. Bansal V, Ryu SY, Lopez N, Allexan S, Krzyzaniak M, Eliceiri B, et al. Vagal stimulation modulates inflammation through a ghrelin mediated mechanism in traumatic brain injury. Inflammation. 2012; 35(1): 214 - 220. https:// doi.org/10.1007/s10753-011-9307-7.

3. Neren D, Johnson MD, Legon W, Bachour SP, Ling G, Divano AA. Vagus nerve stimulation and other neuromodulation methods for treatment of traumatic brain injury. Neurocrit. Care. 2016; 24(2): 308 - 319. https://doi. org/10.1007/s12028-015-0203-0.

4. Ruffoli R, Giorgi FS, Pizzanelli C, Murri L, Paparelli A, Fornai F. The chemical neuroanatomy of vagus nerve stimulation. J Chem Neuroanat. 2011; 42(4): 288 - 296. https:// doi.org/10.1016/j.jchemneu.2010.12.002.

5. Yuan H, Silberstein SD. Vagus nerve and vagus nerve stimulation, a comprehensive review: Part II. Headache. 2016; 56(1): 71 - 8. https:// doi.org/10.1111/head.12647.

6. Kumaria A, Tolias CM. Is there a role for vagus nerve stimulation therapy as a treatment of traumatic brain injury? $B r$ J Neurosurg. 2012; 26(3): 316 - 320. https://doi.org/10.3109/02688 697.2012.663517.

7. Shi C, Flanagan SR, Samadani U. Vagus nerve stimulation to augment recovery from severe traumatic brain injury impeding consciousness: A prospective pilot clinical trial. Neurol Res. 2013; 35(3): 263 - 276. https://doi.org/10.1179 /1743132813Y.0000000167.

8. Clough RW, Neese SL, Tan AA, Duke A, Roosevelt RW, Browning RA, Smith DC. Cortical edema in moderate fluid percussion brain injury is attenuated by vagus nerve stimulation. Neuroscience. 2007; 147(2): 286 - 93. https:// doi.org/10.1016/j.neuroscience.2007.04.043.

9. Godoy DA, Videtta W, Di Napoli M. Practical approach to posttraumatic intracranial 
hypertension according to pathophysiologic reasoning. Neurol Clin. 2017; 35(4): 613 - 640. https://doi.org/10.1016/j.ncl.2017.06.002.

10. Godoy DA, Lubillo S, Rabinstein AA. Pathophysiology and management of intracranial hypertension and tissular brain hypoxia after severe traumatic brain injury. Neurosurg Clin N Am. 2018; 29(2): 195 - 212. https://doi.org/10.1016/j.nec.2017.12.001.

11. Stocchetti N, Maas AIR. Traumatic intracranial hypertension. $N$ Engl J Med. 2014; 370(22): 2121 - 2130. https://doi.org/10.10156/ NEJMra1208708.

12. Arrojo FG, Muñoz AH, Anciones B. Hipertensión intracraneal aguda. Neurología. 2010; 25 (Suppl 1): 3 - 10. https://doi. org/10.1016/S0213-4853(10)70044-X.

13. Lopez NE, Krzyzaniak MJ, Costantini TW, Putnam J, Hageny A-M, Eliceiri B, et al. Vagal nerve stimulation decreases blood-brain barrier disruption after traumatic brain injury. J Trauma Acute Care Surg. 2012; 72(6): 1562 - 1566. https://doi.org/10.1097/TA.0b013e3182569875.

14. Morrison RA, Hulsey DR, Adcock KS, Rennaker II RL, Kilgard MP, Hays SA. Vagus nerve stimulation intensity influences motor cortex plasticity. Brain Stimul. 2019; 12(2): 256 - 262. https://doi.org/10.1016/j.brs.2018.10.017.
15. Cai PY, Bodhit A, Derequito R, Ansari S, Abukhalil F, Thenkabail S, et al. Vagus nerve stimulation in ischemic stroke: Old wine in a new bottle. Front Neurol. 2014; 5: 107. https:// doi.org/10.3389/fneur.2014.00107.

16. Wheless JW, Gienapp AJ, Ryvlin P. Vagus nerve stimulation (VNS) therapy update. Epilepsy Behav. 2018; 88S: 2 - 10. https://doi. org/10.1016/i.yebeh.2018.06.032.

17. George MS, Sackeim HA, Rush AJ, Marangell LB, Nahas Z, Husain MM, et al. Vagus nerve stimulation: A new tool for brain research and therapy. Biol Psychiatry. 2000; 47(4): 287 - 295. https://doi.org/10.1016/s00063223(99)00308-x.

18. Giordano F, Zicca A, Barba C, Guerrini R, Genitori L. Vagus nerve stimulation: Surgical technique of implantation and revision and related morbidity. Epilepsia. 2017; 58 Suppl 1: 85 - 90. https://doi.org/10.1111/epi.13678.

19. Tronnier VM. Vagus nerve stimulation: Surgical technique and complications. Prog Neurol Surg. 2015; 29: 29 - 38. https://doi. org $/ 10.1159 / 000434653$.

20. Pruitt DT, Schmid AN, Kim LJ, Abe CM, Trieu $\mathrm{JL}$, Choua $\mathrm{C}$, et al. Vagus nerve stimulation delivered with motor training enhances recovery of function after traumatic brain injury. 2016; 33(9): 871 - 9. https://doi. org/10.1089/neu.2015.3972.

21. Saulle MF, Schambra HM. Recovery and rehabilitation after intracerebral hemorrhage. Semin Neurol. 2016; 36(3): 306 - 312. https:// doi.org/10.1055/s-0036-1581995.

22. Smith DC, Tan AA, Duke A, Neese SL, Clough RW, Browning RA, Jensen RA. Recovery of function after vagus nerve stimulation initiated 24 hours after fluid percussion brain injury. J Neurotrauma. 20016; 23(10): 1549 - 1560. https://doi.org/10.1089/neu.2006.23.1549.

23. Tubbs RS, Wellons 3rd JC, Blount JP, Oakes WJ. Left-sided vagus nerve stimulation decreases intracranial pressure without resultant bradycardia in the pig: A potential therapeutic modality for humans. Childs Nerv Syst. 2004; 20(5): 309 - 312. https://doi.org/10.1007/ s00381-004-0947-x. 